\title{
The microRNA-429/DUSP4 axis regulates the sensitivity of colorectal cancer cells to nintedanib
}

\author{
GUOHUA CHENG, YARONG LI, ZHAOYU LIU and XIANG SONG \\ Department of Oncology, Second Hospital of Shanxi Medical University, Taiyuan, Shanxi 030001, P.R. China
}

Received August 12,2020; Accepted December 2, 2020

DOI: $10.3892 / \mathrm{mmr} .2021 .11867$

\begin{abstract}
Colorectal cancer (CRC) is recognized as one of the most common malignancies, which ranks third among all cancer-related deaths worldwide. Nintedanib is an orally available tyrosine kinase inhibitor that can treat CRC; however, drug resistance to nintedanib leads to unsatisfactory treatments for patients with CRC. The aim of the present study was to explore whether overexpression of miR-429 elevated the sensitivity of CRC cells to nintedanib by downregulating dual specificity protein phosphatase 4 (DUSP4). The nintedanib-resistant CRC cell model was established via the treatment of cells with nintedanib in a dose-dependent manner. Reverse transcription-quantitative PCR was used to detect the expression levels of miR-429 and DUSP4, and to confirm the transfection efficiency of miR-429 mimic and DUSP4 overexpression plasmid. Cell Counting Kit-8 assay was utilized to measure the inhibition rate of cells. Western blotting was conducted to observe the expression levels of DUSP4 protein, apoptosis-related proteins and proteins related to the JNK signaling pathway. Dual-luciferase reporter assay was performed to evaluate luciferase activity and TUNEL assay was conducted to detect the apoptosis of cells. The results revealed that miR-429 mimic elevated the sensitivity of CRC cells to nintedanib. Moreover, by ENCORI prediction, DUSP4 was identified as a target gene of miR-429, and overexpression of DUSP4 reversed the inducing effect of miR-429 overexpression on the sensitivity of CRC cells to nintedanib. In conclusion, overexpression of miR-429 may elevate the sensitivity of CRC cells to nintedanib through inhibition of the JNK signaling pathway by targeting DUSP4. These findings may aid in the prevention of drug resistance of CRC cells to nintedanib.
\end{abstract}

Correspondence to: Dr Xiang Song, Department of Oncology, Second Hospital of Shanxi Medical University, 382 Wuyi Road, Taiyuan, Shanxi 030001, P.R. China

E-mail: songxiang96@126.com

Key words: microRNA-429, DUSP4, colorectal cancer cells, nintedanib

\section{Introduction}

Colorectal cancer (CRC) is recognized as one of the most common malignancies, ranking third among all causes of cancer-related mortality worldwide $(1,2)$. The available treatment options for CRC in the early stages (I-III) are surgery and chemoradiotherapy. However, $\sim 1 / 4$ of patients are diagnosed with advanced CRC (3). With different stages of the disease being present at the time of diagnosis and with varying techniques used for CRC treatment, the median survival time of patients with $\mathrm{CRC}$ ranges from $<1$ to 5 years (4). Efficient treatment methods and prognostic markers for patients with CRC are currently lacking; therefore, it is important to investigate the underlying mechanism of CRC and to develop effective drugs targeting this disease.

Nintedanib is an orally available tyrosine kinase inhibitor developed to treat numerous types of cancer (5). Nintedanib inhibits the vascular endothelial growth factor receptors (VEGFRs) 1-3, platelet-derived growth factor receptors (PDGFRs) $\alpha$ and $\beta$ and fibroblast growth factor receptors (FGFRs) 1-3, which participate in the pathogenic process of idiopathic pulmonary fibrosis $(6,7)$. Moreover, nintedanib is clinically used for the treatment of hepatic failure, liver cancer, ovarian cancer, prostate cancer and CRC. It has been reported that treatment with nintedanib decreases the risk of acute exacerbations and that it can stabilize disease progression (8).

MicroRNAs (miRNAs/miRs) serve important roles in the development and progression of malignancies. For example, miR-429 has been associated with the regulation of various types of cellular processes (9). It has been reported that miR-429 is downregulated in non-small cell lung cancer cells resistant to nintedanib (10). Moreover, miR-429 may inhibit the invasion and migration of CRC cells by targeting the PAK6/cofilin signaling pathway (11). Using the software ENCORI database (http://starbase.sysu.edu.cn/index.php), preliminary analysis revealed that miR-429 can interact with dual specificity protein phosphatase 4 (DUSP4), which is a member of the dual specificity phosphatase family and specifically dephosphorylates the MAP kinases ERK1/2, p38 and JNK (12,13). In addition, miR-429 has been reported to promote the epithelial-mesenchymal transition of gastric cancer cells to enhance their resistance to adriamycin (14).

The aim of the present study was to investigate whether overexpression of miR-429 can increase the sensitivity of CRC cells to nintedanib by downregulating DUSP4. 


\section{Materials and methods}

Cell culture and construction of resistant cells. The CRC cell lines, SW480, LoVo and HCT116 used in the present study were purchased from the American Type Culture Collection. Cells were cultured in RPMI-1640 medium (Gibco; Thermo Fisher Scientific, Inc.) supplemented with 10\% FBS (Gibco; Thermo Fisher Scientific, Inc.) at $37^{\circ} \mathrm{C}$ with $5 \% \mathrm{CO}_{2}$. These cell lines were amplified and frozen, and one aliquot of each was thawed for subsequent experimentation. All cells were routinely screened for the absence of mycoplasma (10).

CRC cells in logarithmic growth were taken and placed in a cell culture dish at a concentration of $2 \times 10^{6}$ cells $/ \mathrm{ml}$. The cells were treated with $0.01 \mu \mathrm{M}$ nintedanib at $37^{\circ} \mathrm{C}$ for $48 \mathrm{~h}$ and medium was replaced by normal culture solution. After 2 to 3 weeks of continuous culture, the concentration of nintedanib was increased step by step, and CRC cells could grow steadily in $0.1 \mu \mathrm{M}$ nintedanib-containing medium, thus the CRC-resistant to nintedanib (CRC-R) cell line was obtained.

Cell treatment. Cell suspensions $(5,000$ cells/well) were seeded into 96-well plates and treated with increasing concentrations of nintedanib $(0,0.01,0.1,1,10$ and $100 \mu \mathrm{M}$; Selleck Chemicals) at $37^{\circ} \mathrm{C}$ for $72 \mathrm{~h}$ to verify the construction of resistant cells.

Cell transfection. SW480-resistant to nintedanib (SW480-R) cells seeded into a 24 -well plate $\left(5 \times 10^{4} /\right.$ well) were transfected with miR-429 mimic $(100 \mathrm{nM})$ and miR-negative control (miR-NC) (100 nM), and/or DUSP4 overexpression plasmid (Oe-DUSP4; $50 \mathrm{nM}$ ) and the negative control plasmid (Oe-NC; $50 \mathrm{nM}$; Guangzhou RiboBio Co., Ltd.) at $37^{\circ} \mathrm{C}$ for $48 \mathrm{~h}$; untreated cells were set as a control group. DUSP4 sequence was cloned into pcDNA3.1 vector (Invitrogen; Thermo Fisher Scientific, Inc.).

Transfection was performed using Lipofectamine ${ }^{\circledR} 2000$ (Invitrogen; Thermo Fisher Scientific, Inc.) according to the manufacturer's instructions. The miR-429 mimic sequence was 5'-TAATACTGTCTGGTAAAACCGT-3' and the miR-NC sequence was 5'-UUGAGGCUUCAAUCGACGUTT-3'.

Cell Counting Kit-8 (CCK-8) assay. CRC cells and CRC-R cells in logarithmic growth phase were placed in a 96-well plate $\left(10^{3}\right.$ cells/well) and treated with different concentrations of nintedanib $(0.01,0.1,1,10$ and $100 \mu \mathrm{M})$. SW480-R cells in logarithmic growth phase were placed in a 96-well plate $\left(10^{3}\right.$ cells/well) and transfected with mimic NC or miR-429 mimic. After $72 \mathrm{~h}$ of continuous culture, $10 \mu \mathrm{lCCK}-8$ reagent (Dojindo Molecular Technologies, Inc.) was added to the wells and cells were incubated for $72 \mathrm{~h}$ at $37^{\circ} \mathrm{C}$. The absorbance at $450 \mathrm{~nm}$ was determined using a microplate reader (Bio-Rad Laboratories, Inc.). Inhibition rate was calculated as follows: Inhibition rate $(\%)=($ control group - experimental group)/(control group - blank group) x $100 . \mathrm{IC}_{50}$ value was calculated using GraphPad Prism version 6.0 (GraphPad Software, Inc.).

Reverse transcription-quantitative PCR (RT-qPCR) analysis. Total RNA was extracted from CRC cells and CRC-R cells using TRIzol ${ }^{\circledR}$ reagent (Thermo Fisher Scientific, Inc.). Total RNA was then reverse-transcribed into cDNA using a TransScript ${ }^{\circledR}$ First-Strand cDNA Synthesis SuperMix kit (TransGen Biotech Co., Ltd.) according to manufacturer's protocol. qPCR was performed using iTaq ${ }^{\mathrm{TM}}$ Universal SYBR ${ }^{\circledR}$ Green Supermix (Bio-Rad Laboratories, Inc.) on an ABI 7500 instrument (Applied Biosystems; Thermo Fisher Scientific, Inc.). PCR thermocycling conditions were $90 \mathrm{sec}$ at $95^{\circ} \mathrm{C}, 30 \mathrm{sec}$ at $95^{\circ} \mathrm{C}$, $20 \mathrm{sec}$ at $65^{\circ} \mathrm{C}$ and $30 \mathrm{sec}$ at $72^{\circ} \mathrm{C}$, for 40 cycles. Primers were miR-429 forward, 5'-GGGGGTAATACTGTCTGGT-3' and reverse, 5'-TGCGTGTCGTGGAGTC-3'; U6 forward, 5'-GCTTCGGCAGCACATATACTAA-3' and reverse, 5'-CGAATTTGCGTGTCATCCTT-3'; DUSP4 forward, 5'-TCACGGCTCTGTTGAATGTC-3' and reverse, 5'-GATGTCGGCCTTGTGGTTAT-3'; GAPDH forward, 5'-CGAATTTGCGTGTCATCCTT-3' and reverse, 5'-CGAATTTGCGTGTCATCCTT-3'. The relative expression levels of miRNA or mRNA were normalized to U6 or GAPDH, and were calculated based on the $2^{-\Delta \Delta C q}$ method (15).

Western blotting. Cellular proteins were extracted from CRC cells and CRC-R cells lysed using lysis buffer (CWBio). Protein concentration was quantified by BCA assay. Equal amounts of protein $(20 \mu \mathrm{g})$ were separated by SDS-PAGE (Beyotime Institute of Biotechnology) on 10\% gels and were then electrotransferred onto polyvinylidene difluoride (PVDF) membranes (EMD Millipore). After blocking in Tris-buffered saline-Tween-20 (0.05\%) containing 5\% non-fat dry milk for $1 \mathrm{~h}$ at room temperature, the membranes were incubated with primary antibodies overnight at $4^{\circ} \mathrm{C}$. Membranes were then incubated with horseradish peroxidase-conjugated anti-rabbit $\operatorname{IgG}$ secondary antibody (cat. no. 7074; 1:1,000; Cell Signaling Technology, Inc.) for the detection of primary antibodies at room temperature for $2 \mathrm{~h}$, and the bands were visualized using an enhanced chemiluminescence detection kit (EMD Millipore) and analyzed by ImageJ software (v.1.52; National Institutes of Health). The following primary antibodies were used: Bcl-2 (cat. no. ab32124; 1:1,000; Abcam), Bax (cat. no. ab182734; 1:1,000; Abcam), cleaved caspase-3 (cat. no. ab2302; 1:1,000; Abcam), caspase-3 (cat. no. ab13847; 1:1,000; Abcam), phosphorylated (p)-JNK (cat. no. ab215208; 1:1,000; Abcam), c-Jun (cat. no. ab40766; 1:1,000; Abcam), JNK (cat. no. ab199380; 1:1,000; Abcam), multi-drug resistance protein (MDR1; cat. no. 13342; 1:1,000; Cell Signaling Technology, Inc.), DUSP4 (cat. no. ab216576; 1:1,000; Abcam) and GAPDH (cat. no. ab9485; 1:1,000; Abcam).

TUNEL assay. SW480 cells, SW480-R cells and the corresponding transfected cells were seeded into a 24 -well plate $\left(5 \times 10^{4} /\right.$ well) and cultured at $37^{\circ} \mathrm{C}$ with $1 \mu \mathrm{M}$ nintedanib for $72 \mathrm{~h}$. After the supernatant was discarded, cells were washed with PBS, fixed with $4 \%$ paraformaldehyde for $20 \mathrm{~min}$ at room temperature and washed three more times with PBS. Cells were treated with $75 \%$ ethanol at $4{ }^{\circ} \mathrm{C}$ overnight, and were washed once with PBS the following day. According to the manufacturer's instructions of the TUNEL apoptosis kit (Roche Diagnostics), apoptosis was observed using a fluorescence microscope (magnification, x200; Olympus Corporation).

Dual-luciferase reporter assay. ENCORI database (http:// starbase.sysu.edu.cn/index.php) predicted that DUSP4 could 
A

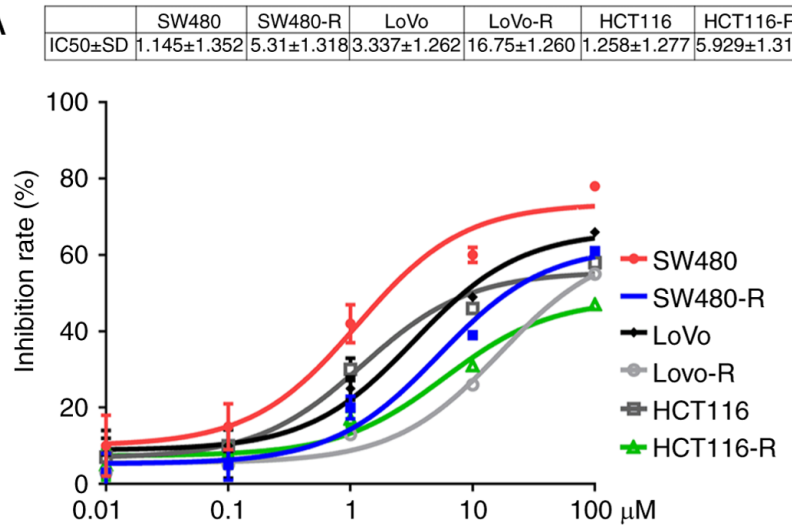

B

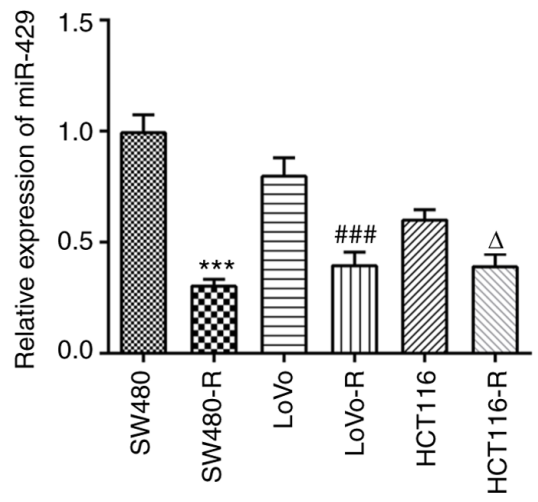

Figure 1. Construction of a nintedanib-resistant colorectal cancer cell model. (A) $\mathrm{IC}_{50}$ value and inhibition rate of cells treated with different concentrations of nintedanib, as determined by Cell Counting Kit-8 assay. (B) Expression levels of miR-429 in CRC cell lines, as determined by reverse transcriptionquantitative PCR. ${ }^{* * *} \mathrm{P}<0.001$ vs. SW480; ${ }^{\# \#} \mathrm{P}<0.001$ vs. LoVo; ${ }^{\Delta} \mathrm{P}<0.05$ vs. HCT116. miR-429, microRNA-429; -R, resistant to nintedanib.

A

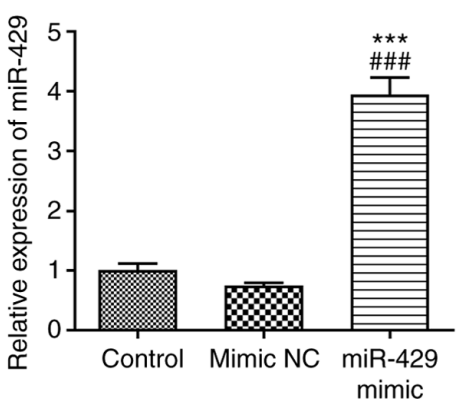

B
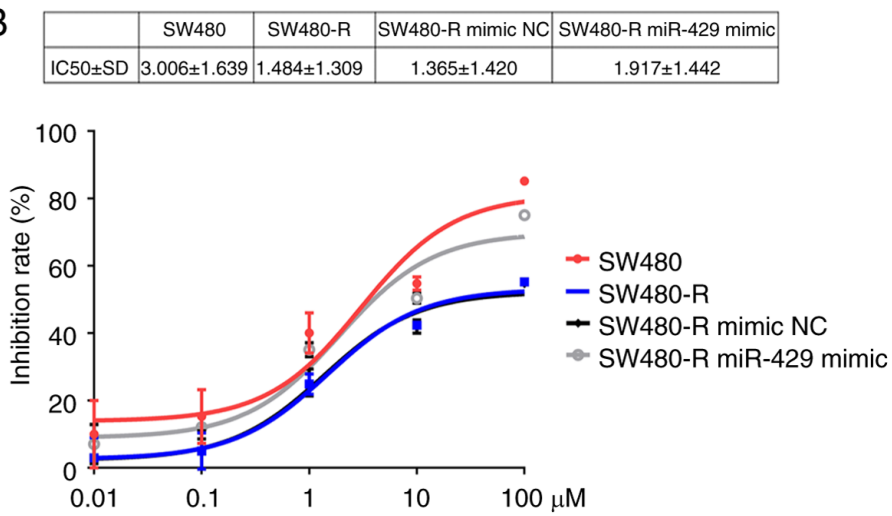

Figure 2. miR-429 mimic elevates the inhibition rate of resistant colorectal cancer cells treated with nintedanib. (A) Transfection efficiency of the miR-429

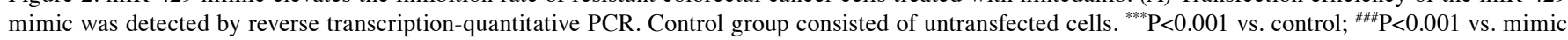
NC. (B) $\mathrm{IC}_{50}$ value and inhibition rate of cells treated with different concentrations of nintedanib post-transfection, as determined by Cell Counting Kit- 8 assay. miR-429, microRNA-429; NC, negative control; -R, resistant to nintedanib.

combine with miR-429. DUSP4 3'-untranslated region luciferase reporter gene plasmid was constructed by Shanghai GenePharma Co., Ltd. DUSP4 mutation was constructed using the Quick-Change Site-Directed Mutagenesis kit (Agilent Technologies, Inc.). DUSP4-wild-type (WT) and DUSP4mutant (MUT) plasmids were generated via subcloning downstream of the luciferase vector. SW480 cells were seeded into a 24 -well plate $\left(5 \times 10^{4} /\right.$ well $)$ and co-transfected with miR-429 mimic (100 nM) + DUSP4-WT $(50 \mathrm{nM})$ or miR-429 mimic (100 nM) + DUSP4-MUT (50 nM) using Lipofectamine ${ }^{\circledR} 2000$ (Invitrogen; Thermo Fisher Scientific, Inc.) at $37^{\circ} \mathrm{C}$. After $48 \mathrm{~h}$, cells were assessed using the DualLuciferase Reporter Gene Assay kit (Beyotime Institute of Biotechnology) according to the manufacturer's instructions. Luminescent signals were quantified with a luminometer (Centro XS3 LB 960; Titertek-Berthold), and firefly luciferase activity was normalized to Renilla luciferase activity.

Statistical analysis. Data are presented as the mean \pm SD obtained from three independent experiments. Statistical analysis was conducted using GraphPad Prism version 6.0 (GraphPad Software, Inc.). Statistical comparisons among multiple groups were performed using one-way ANOVA followed by Tukey's post hoc test. $\mathrm{P}<0.05$ was considered to indicate a statistically significant difference.

\section{Results}

Establishment of nintedanib-resistant CRC cells. The inhibition rate of cells treated with different concentrations of nintedanib was measured using a CCK-8 assay. It was observed that the inhibition rate of SW480 cells pretreated with nintedanib for $72 \mathrm{~h}$ was decreased to a larger extent compared with the other cells lines and the $\mathrm{IC}_{50}$ of SW480 cells was $1.145 \pm 1.352$ (Fig. 1A). Moreover, miR-429 expression was significantly downregulated in the SW480-R cells compared with SW480 cells (Fig. 1B). Thus, SW480-R and SW480 cells were chosen for the subsequent experiments. SW480-R cells were resistant to nintedanib, whereas SW480 cells were not.

miR-429 mimic increases the sensitivity of resistant CRC cells to nintedanib. After transfection with the miR-429 mimic, the successful transfection efficiency of miR-429 mimic was confirmed via RT-qPCR (Fig. 2A). After treatment of the cells with different concentrations of nintedanib, SW480-R cells transfected with the miR-429 mimic demonstrated an elevated 


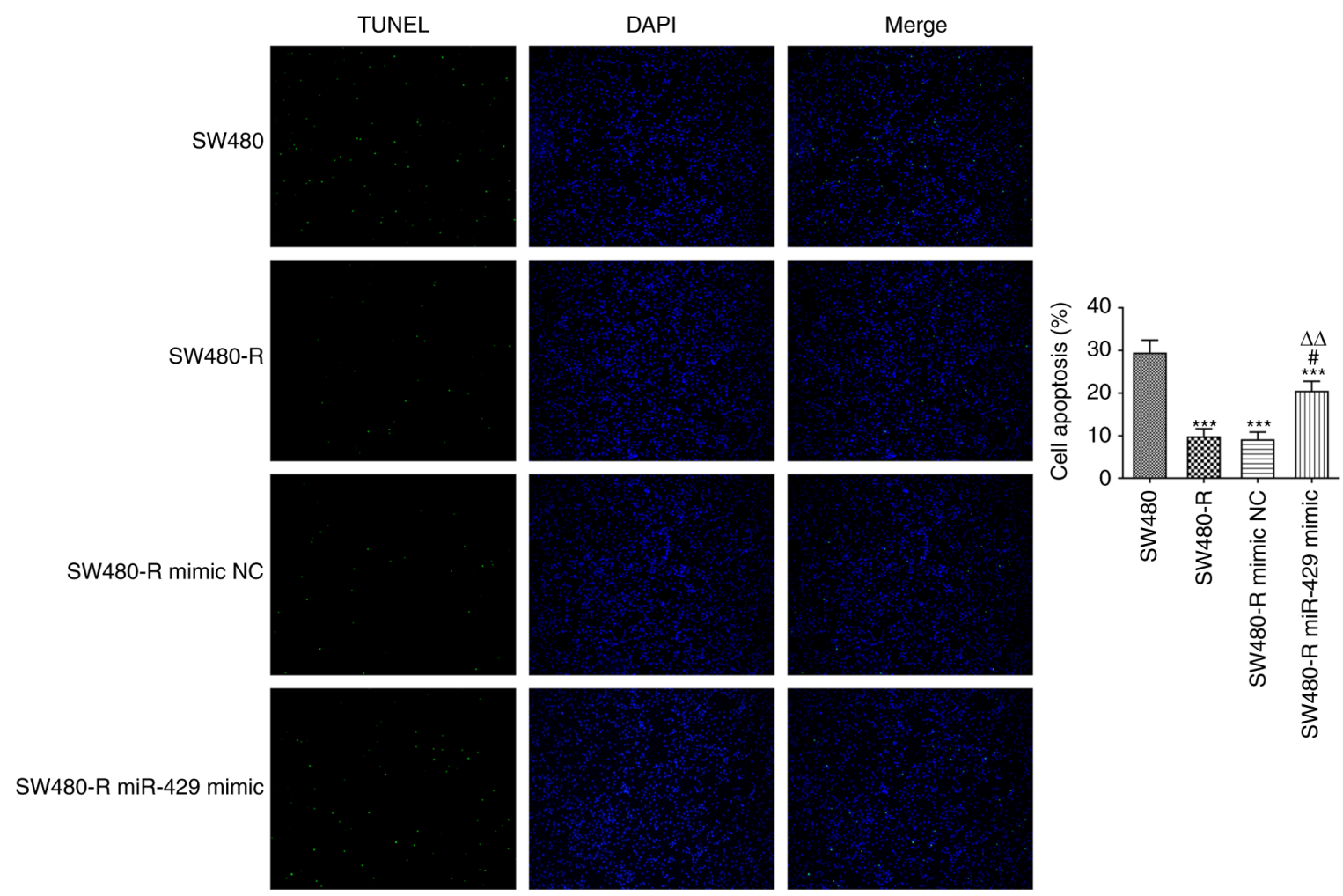

Figure 3. miR-429 mimic promotes the apoptosis of resistant colorectal cancer cells treated with nintedanib. Cell apoptosis affected by nintedanib was detected by TUNEL assay (magnification, $\mathrm{x} 200$ ). ${ }^{* * * *} \mathrm{P}<0.001$ vs. SW480; ${ }^{*} \mathrm{P}<0.05$ vs. SW480-R; ${ }^{\Delta \Delta} \mathrm{P}<0.01$ vs. SW480-R mimic NC. miR-429, microRNA-429; $\mathrm{NC}$, negative control; - $\mathrm{R}$, resistant to nintedanib.

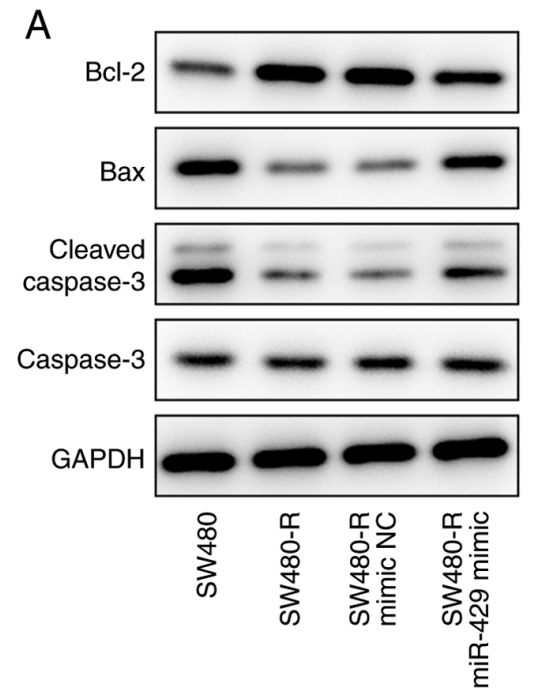

$\mathrm{B}$.
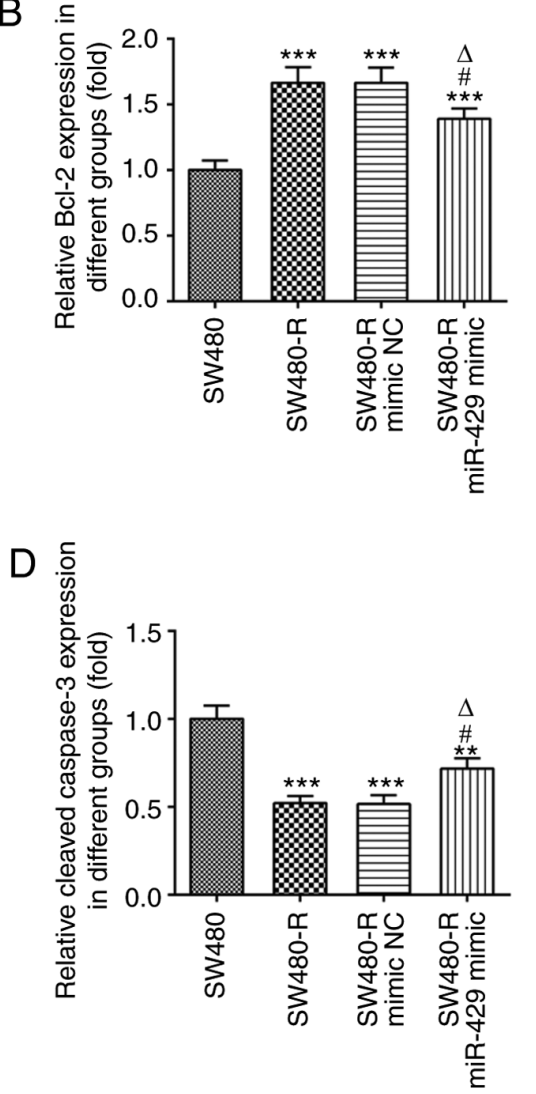

$\mathrm{C} \subseteq$

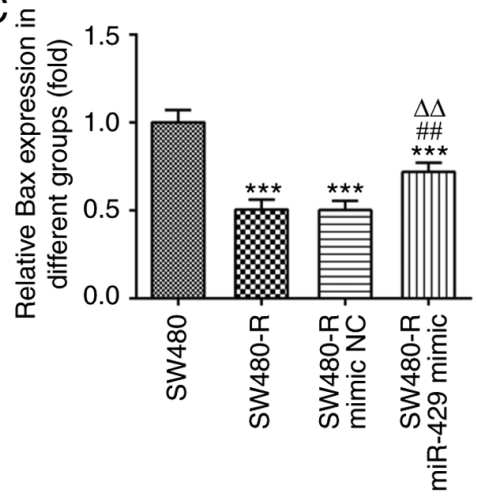

E

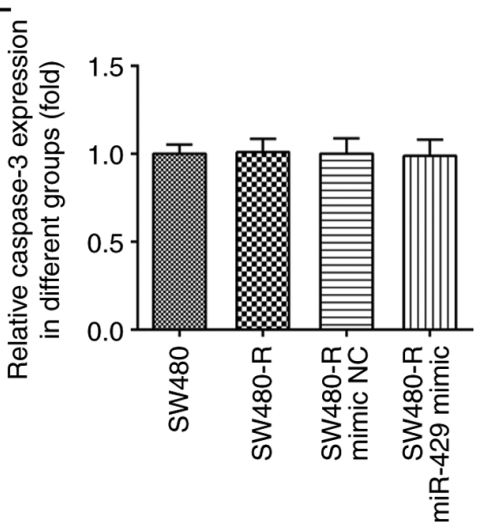

Figure 4. miR-429 mimic affects the expression levels of apoptosis-related proteins in resistant CRC cells treated with nintedanib. (A) Western blotting of apoptosis-related proteins in resistant CRC cells treated with nintedanib. The expression levels of apoptosis-related proteins, including (B) Bcl-2, (C) Bax, (D) cleaved caspase-3 and (E) caspase-3 in CRC cells were detected by western blotting. ${ }^{* *} \mathrm{P}<0.01,{ }^{* * * *} \mathrm{P}<0.001$ vs. SW480; ${ }^{*} \mathrm{P}<0.05$, ${ }^{\# \#} \mathrm{P}<0.01$ vs. SW480-R; ${ }^{\Delta} \mathrm{P}<0.05,{ }^{\Delta} \mathrm{P}<0.01$ vs. SW480-R mimic NC. CRC, colorectal cancer; miR-429, microRNA-429; NC, negative control; - $\mathrm{R}$, resistant to nintedanib. 

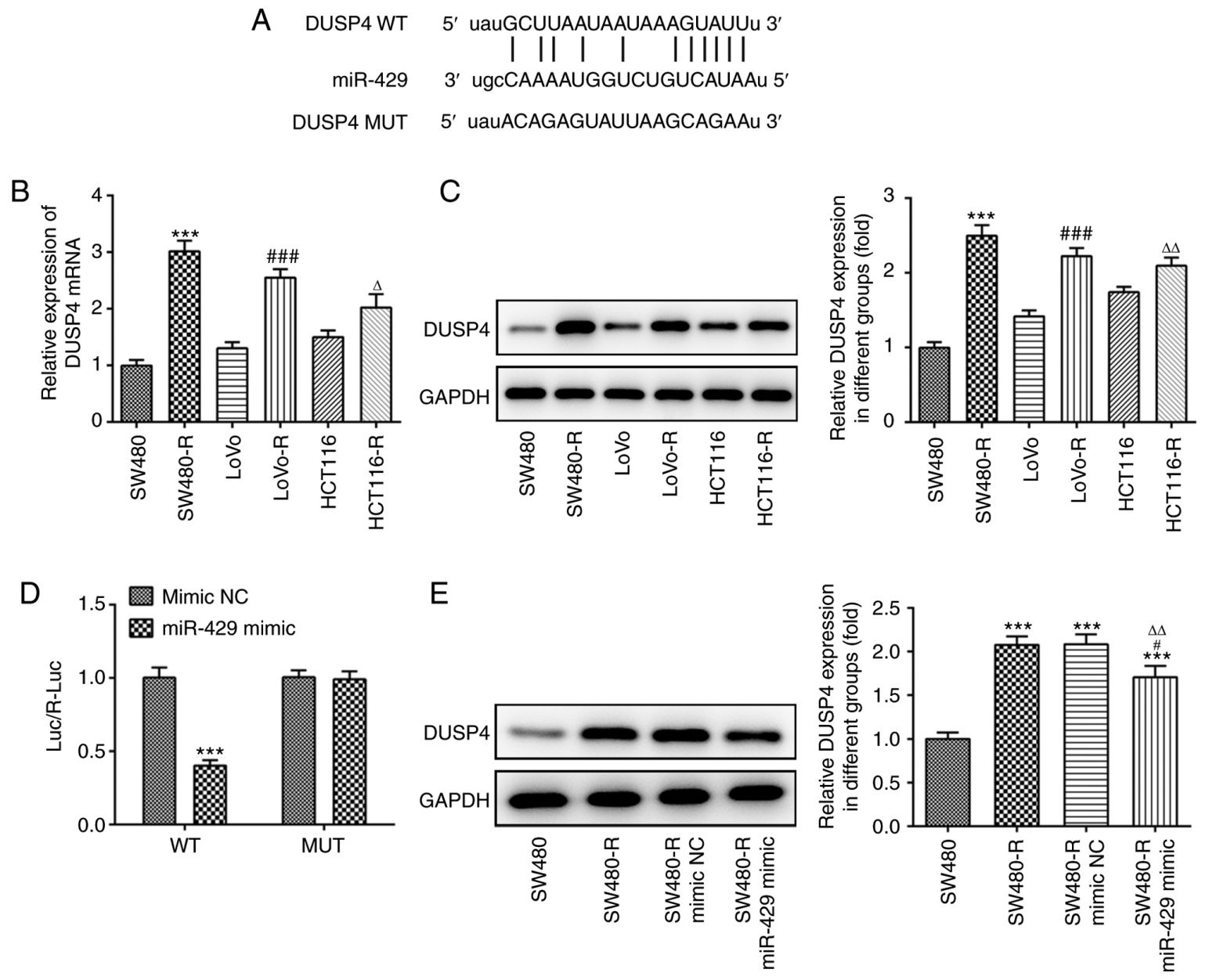

Figure 5. DUSP4 is a direct target gene of miR-429. (A) Binding sites between miR-429 and DUSP4 predicted by ENCORI. (B) mRNA expression levels of DUSP4 in CRC cells and resistant CRC cells, as determined by reverse transcription-quantitative PCR. ${ }^{* * *} \mathrm{P}<0.001$ vs. SW480; ${ }^{* \# \#} \mathrm{P}<0.001 \mathrm{vs}$. LoVo; ${ }^{\wedge} \mathrm{P}<0.05$ vs. HCT116. (C) Protein expression levels of DUSP4 in CRC cells and resistant CRC cells were detected by western blotting. ${ }^{* * *} \mathrm{P}<0.001 \mathrm{vs}$. SW480; ${ }^{* \# \#} \mathrm{P}<0.001$ vs. LoVo; ${ }^{\Delta \Delta} \mathrm{P}<0.01$ vs. HCT116. (D) Detection of luciferase activity. ${ }^{* * * *} \mathrm{P}<0.001$ vs. mimic NC. (E) Protein expression levels of DUSP4 in resistant CRC cells post-transfection, as determined by western blotting. ${ }^{* * *} \mathrm{P}<0.001$ vs. SW480; ${ }^{*} \mathrm{P}<0.05$ vs. SW480-R; ${ }^{\Delta \Lambda} \mathrm{P}<0.01$ vs. SW480-R mimic NC. CRC, colorectal cancer; miR-429, microRNA-429; DUSP4, dual specificity protein phosphatase 4; WT, wild-type; MUT, mutant; NC, negative control; -R, resistant to nintedanib.

inhibition rate compared with SW480-R cells transfected with mimic NC (Fig. 2B).

To further confirm the role of miR-429 in SW480-R cells, cell apoptosis was evaluated. It was revealed that the apoptosis of SW480-R cells was decreased compared with that of SW-480 cells, and the miR-429 mimic increased the apoptosis of SW480-R cells (Fig. 3). Western blotting of apoptosis-related proteins is shown in Fig. 4A. The protein expression levels of Bcl-2 were increased, whereas the expression levels of Bax and cleaved caspase- 3 were decreased in SW480-R cells, and the miR-429 mimic reversed the expression of these proteins (Fig. 4B-D). Caspase-3 expression was not markedly changed in the four groups (Fig. 4E). Therefore, it was suggested that the miR-429 mimic may promote the sensitivity of CRC cells to nintedanib.

DUSP4 is a target of $m i R-429$. ENCORI software was used to predict miR-429 target genes; binding sites between miR-429 and DUSP4 were identified (Fig. 5A). Subsequently, western blotting and RT-qPCR were used to detect the expression levels of DUSP4. DUSP4 expression was increased to a greater extent in SW480-R cells compared with the other cell lines (Fig. 5B and C). Moreover, compared with the mimic $\mathrm{NC}+$ DUSP4-WT group, the luciferase reporter activity of the miR-429 mimic + DUSP4-WT group was decreased (Fig. 5D). Western blotting was performed to measure the expression levels of DUSP4. The results demonstrated that the SW480-R group exhibited increased expression of DUSP4 compared with the SW480 group, and that SW480-R cells transfected with the miR-429 mimic exhibited decreased expression levels of DUSP4 compared with the SW480-R mimic NC group (Fig. 5E). These findings indicated that the expression of DUSP4 was inversely associated with that of miR-429.

Overexpression of DUSP4 reverses the effect of miR-429 overexpression on the sensitivity of $C R C$ cells resistant to nintedanib. To assess whether DUSP4 expression can affect the sensitivity of CRC cells to nintedanib, Oe-DUSP4 was constructed. The results of RT-qPCR and western blotting confirmed the successful transfection of cells with Oe-DUSP4 (Fig. 6A and B). Subsequently, a CCK-8 assay was used to detect the inhibition rate of SW480-R cells pretreated with different concentrations of nintedanib for $72 \mathrm{~h}$. The miR-429 mimic increased the inhibition rate of 
A

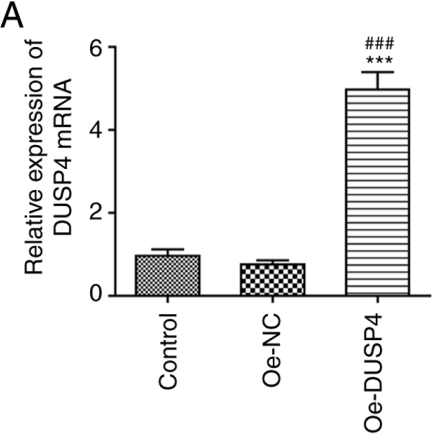

B

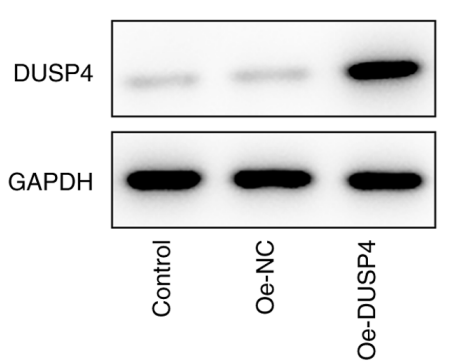

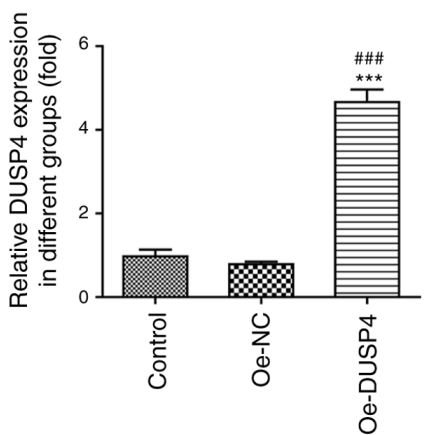

C
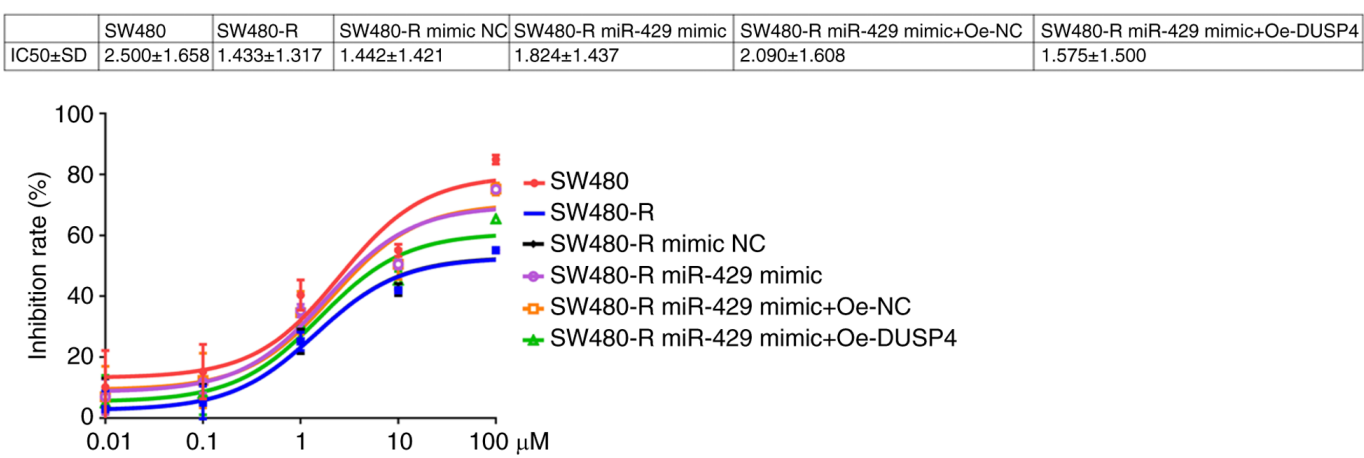

Figure 6. Overexpression of DUSP4 reverses the effect of miR-429 overexpression on the inhibition rate of resistant CRC cells treated with nintedanib. (A) Transfection efficiency of Oe-DUSP4, as determined by reverse transcription-quantitative $\mathrm{PCR}$. ${ }^{* * *} \mathrm{P}<0.001$ vs. control; ${ }^{\# \# \prime} \mathrm{P}<0.001$ vs. Oe-NC. (B) Transfection efficiency of Oe-DUSP4, as determined by western blotting. ${ }^{* * *} \mathrm{P}<0.001$ vs. Control; ${ }^{\# \#} \mathrm{P}<0.001$ vs. Oe-NC. Control group consisted of untransfected cells. (C) $\mathrm{IC}_{50}$ value and inhibition rate of resistant CRC cells treated with different concentrations of nintedanib post-transfection, as determined by Cell Counting Kit-8 assay. CRC, colorectal cancer; miR-429, microRNA-429; DUSP4, dual specificity protein phosphatase 4; NC, negative control; Oe-DUSP4, DUSP4 overexpression plasmid; Oe-NC, NC overexpression plasmid; - $\mathrm{R}$, resistant to nintedanib.

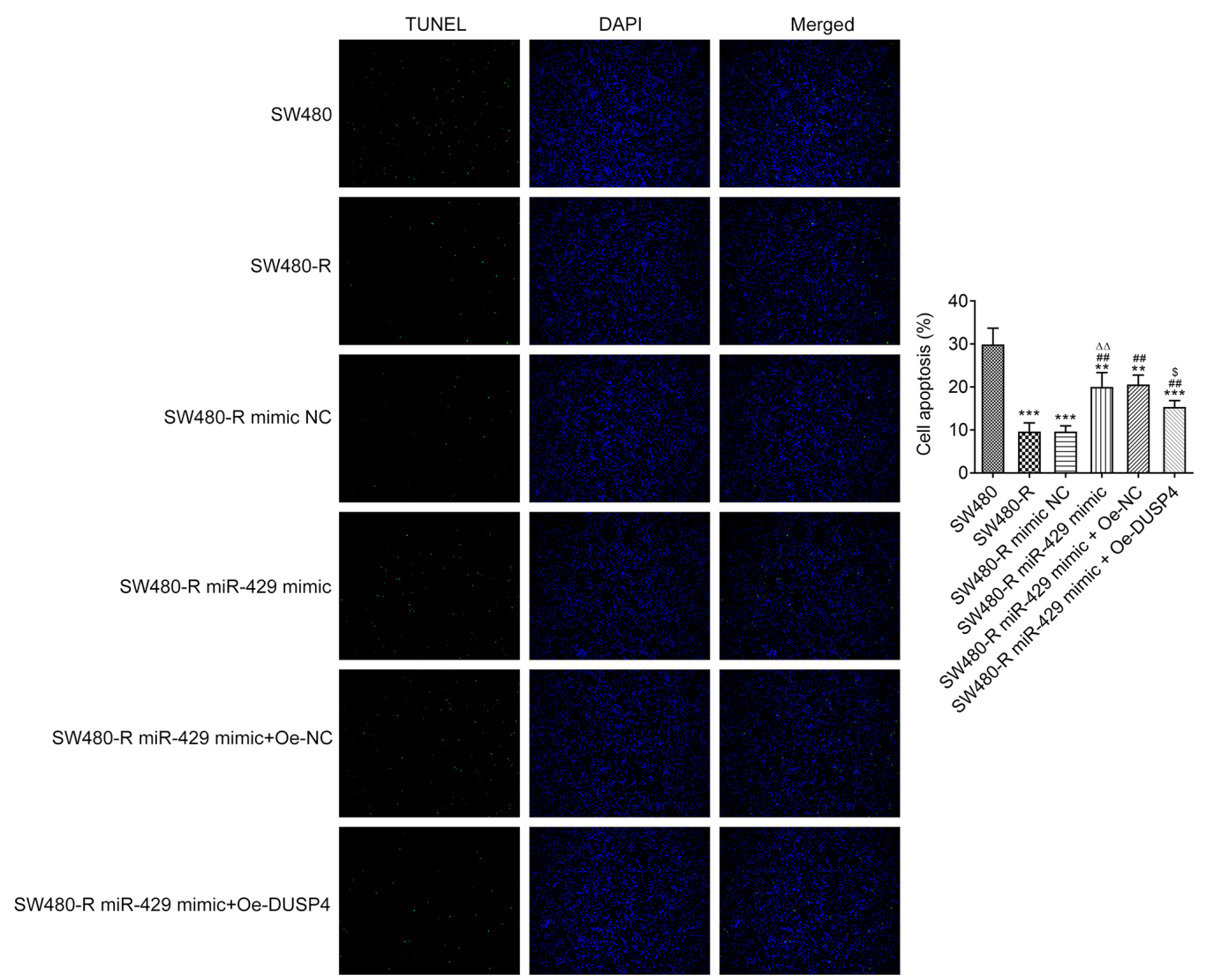

Figure 7. Overexpression of DUSP4 reverses the inductive effect of miR-429 overexpression on the apoptosis of resistant colorectal cancer cells treated with nintedanib. Cell apoptosis affected by nintedanib was detected by TUNEL assay (magnification, $\mathrm{x} 200) .{ }^{* *} \mathrm{P}<0.01,{ }^{* * * *} \mathrm{P}<0.001$ vs. SW480; ${ }^{\# \#} \mathrm{P}<0.01$ vs. SW480-R; ${ }^{\Delta} \mathrm{P}<0.01$ vs. SW480-R mimic NC; ${ }^{\$} \mathrm{P}<0.05$ vs. SW480-R miR-429 mimic + Oe-NC. miR-429, microRNA-429; DUSP4, dual specificity protein phosphatase 4; NC, negative control; Oe-DUSP4, DUSP4 overexpression plasmid; Oe-NC, NC overexpression plasmid; -R, resistant to nintedanib. 


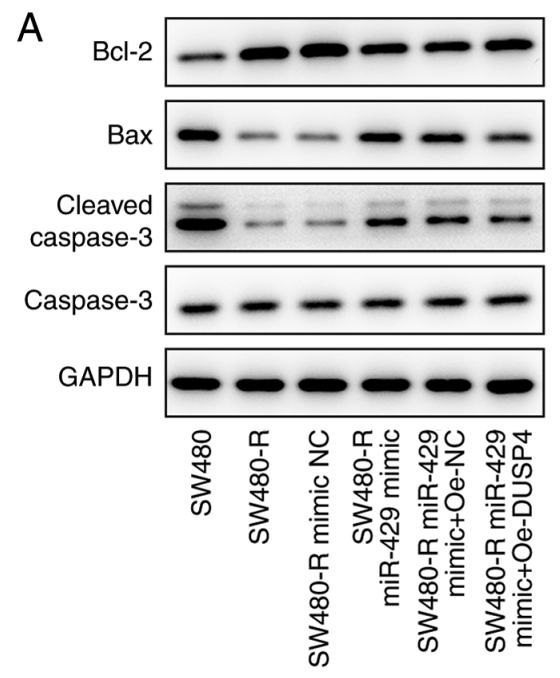

B
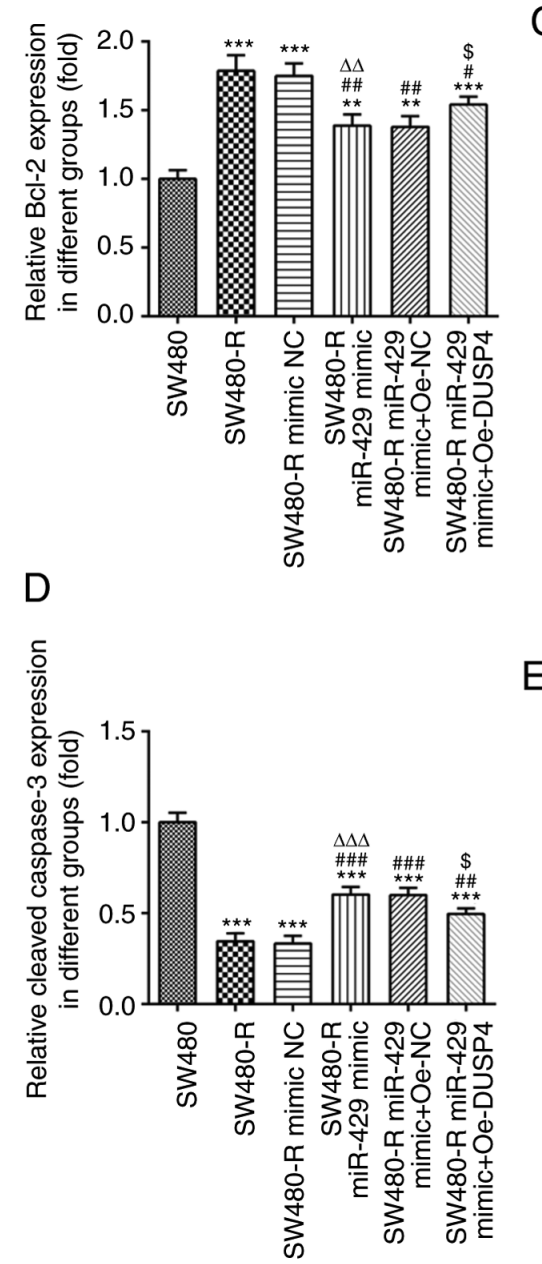

C

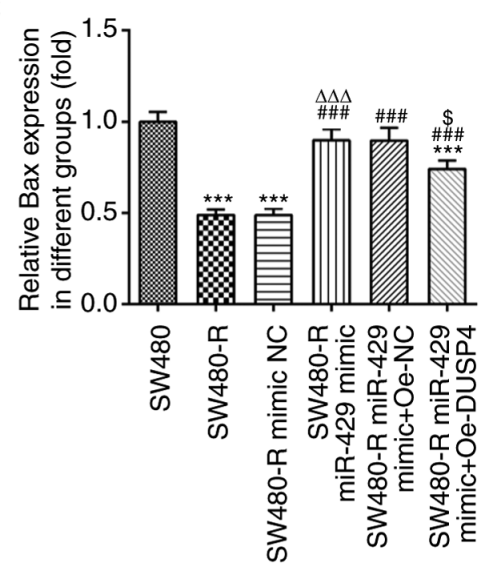

$\mathrm{E}$

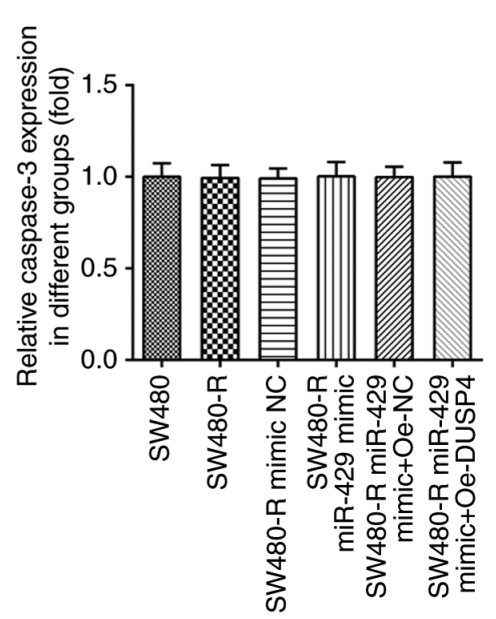

Figure 8. Overexpression of DUSP4 reverses the inductive effect of miR-429 overexpression on the expression of apoptosis-related proteins in resistant CRC cells treated with nintedanib. (A) Western blotting of apoptosis-related proteins in resistant CRC cells treated with nintedanib. The expression levels of apoptosisrelated proteins, including (B) Bcl-2, (C) Bax, (D) cleaved caspase-3 and (E) caspase-3 in CRC cells were detected by western blotting. ${ }^{* * *} \mathrm{P}<0.01,{ }^{* * * *} \mathrm{P}<0.001$ vs. SW480; ${ }^{\# P} \mathrm{P}<0.05,{ }^{\# \#} \mathrm{P}<0.01,{ }^{\# \# \#} \mathrm{P}<0.001$ vs. SW480-R; ${ }^{\Delta \Delta} \mathrm{P}<0.01,{ }^{\Delta \Delta \Delta} \mathrm{P}<0.001$ vs. SW480-R mimic NC; ${ }^{\$} \mathrm{P}<0.05$ vs. SW480-R miR-429 mimic + Oe-NC. CRC, colorectal cancer; miR-429, microRNA-429; DUSP4, dual specificity protein phosphatase 4; NC, negative control; Oe-DUSP4, DUSP4 overexpression plasmid; Oe-NC, $\mathrm{NC}$ overexpression plasmid; -R, resistant to nintedanib.

SW480-R cells (Fig. 6C). Compared with the SW480-R miR-429 mimic + Oe-NC group, SW480-R cells transfected with miR-429 mimic and Oe-DUSP4 exhibited a decreased inhibition rate. The apoptosis of SW480-R cells and the expression levels of apoptosis-related proteins were also evaluated. Overexpression of DUSP4 decreased the apoptosis of miR-429 mimic-transfected SW480-R cells (Fig. 7). Western blotting of apoptosis-related proteins is shown in Fig. 8A. Overexpression of DUSP4 increased the expression levels of $\mathrm{Bcl}-2$, and decreased the expression levels of Bax and cleaved caspase-3 in SW480-R cells transfected with miR-429 mimic (Fig. 8B-D). Caspase-3 expression was not obviously changed in the six groups (Fig. 8E).

It has been reported that DUSP4 can dephosphorylate the MAP kinases ERK1/2, p38 and JNK (12). Thus, western blotting was performed to detect the expression levels of proteins associated with the JNK signaling pathway (Fig. 9A). Oe-DUSP4 significantly decreased the protein expression levels of p-JNK, p/t-JNK and the JNK inhibitor c-Jun compared with those in SW480-R cells transfected with the miR-429 mimic (Fig. 9B, D and E), but increased the expression levels of MDR1 (Fig. 9F). There was no significant difference of JNK expression between the six groups (Fig. 9C). These findings suggested that overexpression of DUSP4 may reverse the inducing effect of miR-429 overexpression on the sensitivity of CRC cells to nintedanib and inhibit the JNK signaling pathway.

\section{Discussion}

Nintedanib, an intracellular inhibitor of tyrosine kinases, such as FGFR, PDGFR and VEGFR, has been approved for the treatment of idiopathic pulmonary fibrosis and various types of cancer $(16,17)$. However, nintedanib resistance often leads to unsatisfactory efficacy in the treatment of diseases and the effect of nintedanib monotherapy on patients with various diseases is limited (18). It is widely known that drug resistance is a major limitation to effective disease treatment, and numerous miRNAs have been reported to be associated with chemoresistance and poor prognosis $(10,19)$. Thus, the present study investigated the association between miRNA expression and drug sensitivity. Notably, a previous study reported 
A

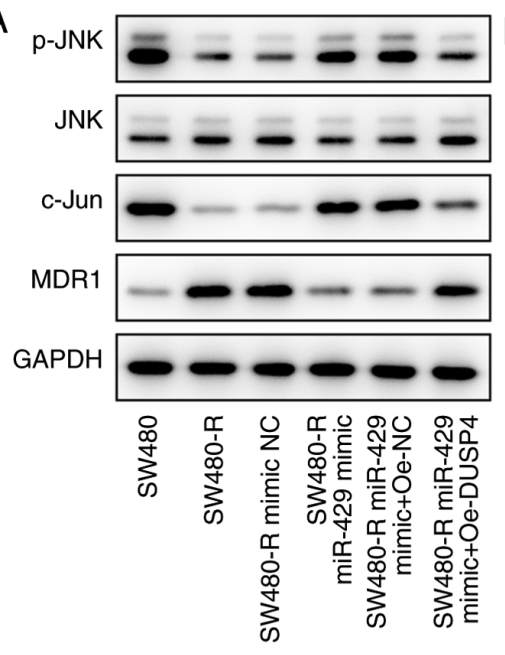

$B$

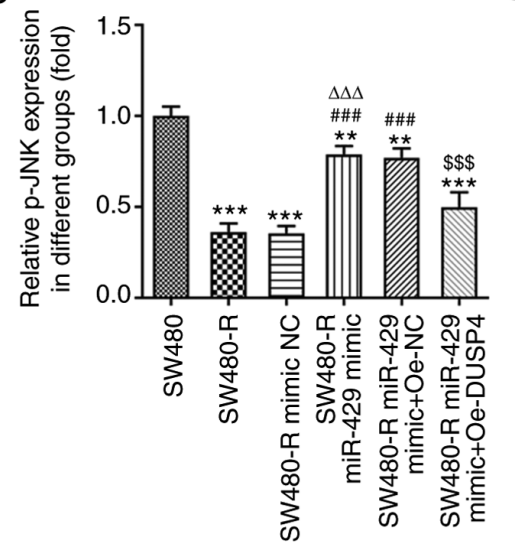

C

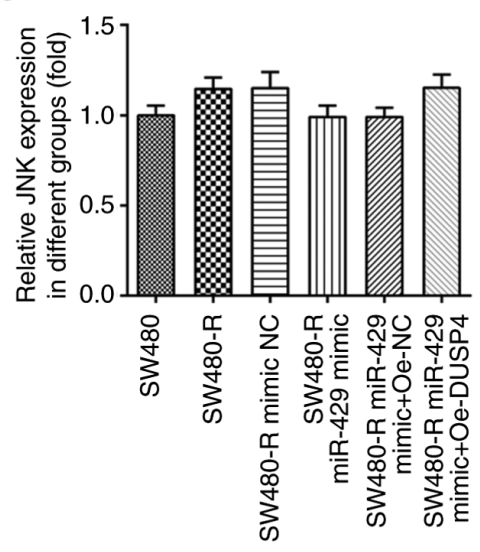

$\mathrm{D}$

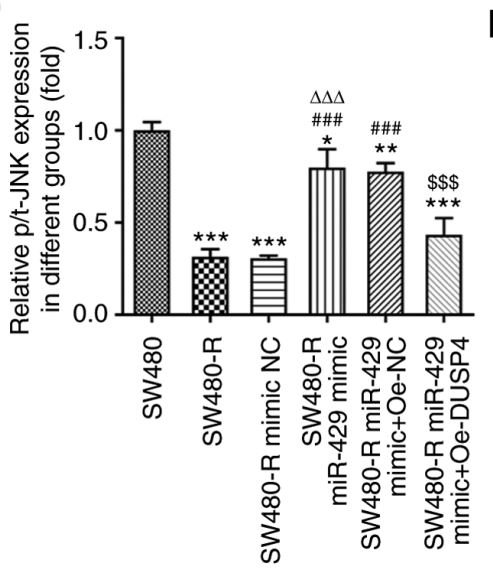

$E$

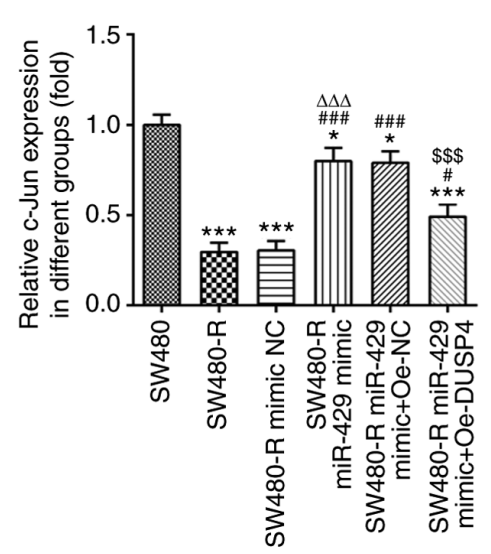

$\mathrm{F}$

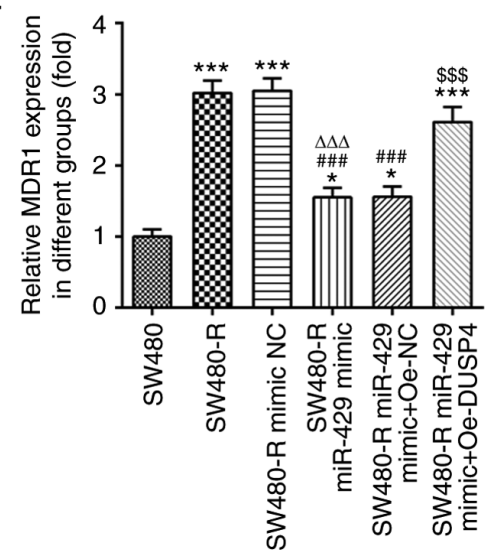

Figure 9. Overexpression of DUSP4 reverses the inductive effect of miR-429 overexpression on the expression of JNK-related proteins in resistant colorectal cancer cells treated with nintedanib. (A) Western blotting of JNK-related proteins. Expression levels of JNK-related proteins, including (B) p-JNK, (C) JNK, (D) p/t-JNK, (E) c-Jun and (F) MDR1 were detected by western blotting. ${ }^{*} \mathrm{P}<0.05,{ }^{* * *} \mathrm{P}<0.01,{ }^{* * * *} \mathrm{P}<0.001$ vs. SW480; ${ }^{\#} \mathrm{P}<0.05,{ }^{\# \# \#} \mathrm{P}<0.001$ vs. SW480-R; ${ }^{\Delta \Delta \Delta} \mathrm{P}<0.001$ vs. SW480-R mimic NC; ${ }^{\$ \$ \$} \mathrm{P}<0.001$ vs. SW480-R miR-429 mimic + Oe-NC. miR-429, microRNA-429; DUSP4, dual specificity protein phosphatase 4; NC, negative control; Oe-DUSP4, DUSP4 overexpression plasmid; Oe-NC, NC overexpression plasmid; -R, resistant to nintedanib; p-, phosphorylated; t-, total; MDR1, multidrug resistance protein.

that the low expression levels of miR-200b and miR-141 were associated with the resistance of non-small cell lung cancer cells to nintedanib (10).

miRNAs have been discovered in multiple organisms and are considered participants in the regulation of gene expression (20). In addition to their involvement in various biological processes, miRNAs may act as oncogenes or tumor suppressors to facilitate or delay tumor progression $(21,22)$. miR-429 is a member of the miR-200 family, which has been reported to serve a key role as a tumor suppressor in numerous types of carcinoma, such as bladder cancer, gastric cancer and hepatocellular carcinoma (23-25). Moreover, miR-429 was shown to be downregulated in metastatic lesions and act as a tumor suppressor in nasopharyngeal carcinoma (26). However, two previous studies reported a contradictory role of miR-429 to that found in the present study; in these previous studies, miR-429 promoted the progression of CRC and non-small cell lung cancer $(27,28)$. In the present study, it was identified that miR-429 increased the sensitivity of SW480 cells to nintedanib and promoted the apoptosis of these cells, demonstrating the potential of miR-429 in the treatment of CRC. Moreover, miR-429 has been shown to be negatively corre- lated with metastatic potential in nasopharyngeal carcinoma cell lines (29).

It was predicted by ENCORI that DUSP4 and miR-429 shared binding sites. Moreover, the present results confirmed that the expression levels of DUSP4 and miR-429 were inversely associated. Subsequent experiments further indicated that DUSP4 may reverse the promoting effect of miR-429 on the sensitivity of SW480 cells to nintedanib, as cells transfected with both the miR-429 mimic and Oe-DUSP4 displayed a lower inhibition rate and apoptotic rate compared with those transfected with the miR-429 mimic only.

It has been reported that most DUSPs negatively regulate the JNK signaling pathway via dephosphorylation (30). Moreover, JNK was previously reported to enhance the catalytic ability of DUSP4, and the expression of DUSP4 has been shown to be upregulated in malignant tissues but downregulated in healthy tissues $(31,32)$. The JNK signaling pathway is closely associated with a number of diseases, particularly cancer. The activity of the JNK signaling pathway is high in most cancer cell lines, and depletion of individual JNKs can inhibit tumorigenesis $(33,34)$. Thus, in recent years, JNKs have been increasingly identified as effective molecular targets for the 
treatment of various malignancies, and the use of a number of JNK inhibitors has been considered as an effective treatment option for tumors $(33,35)$. However, some previous studies have proposed that JNK proteins are implicated in tumor suppression $(33,36)$. Based on this dispute, in the present study, DUSP4 was overexpressed in SW480 cells to examine whether it acted as an inhibitory or enhancing molecule of the JNK signaling pathway. The present results suggested that DUSP4 dephosphorylated JNK, thereby inhibiting the JNK signaling pathway.

In conclusion, the present study identified the role of the miR-429/DUSP4 axis in regulating the sensitivity of CRC cells to nintedanib. Moreover, overexpression of miR-429 may increase the sensitivity of CRC cells to nintedanib through inhibiting the JNK signaling pathway by downregulating DUSP4. These findings may enable the development of prevention strategies for the resistance of CRC cells to nintedanib.

\section{Acknowledgements}

Not applicable.

\section{Funding}

No funding was received.

\section{Availability of data and materials}

The datasets used and/or analyzed during the current study are available from the corresponding author on reasonable request.

\section{Authors' contributions}

XS contributed to the conception and design of this study. GC performed the experiments, collected the data and performed statistical analysis with the help of YL and ZL. GC drafted the manuscript, which was corrected and revised by XS. All authors read and approved the final manuscript.

\section{Ethics approval and consent to participate}

Not applicable.

\section{Patient consent for publication}

Not applicable.

\section{Competing interests}

The authors declare that they have no competing interests.

\section{References}

1. Siegel RL, Miller KD and Jemal A: Cancer statistics, 2019. CA Cancer J Clin 69: 7-34, 2019.

2. Fanali C, Lucchetti D, Farina M, Corbi M, Cufino V, Cittadini A and Sgambato A: Cancer stem cells in colorectal cancer from pathogenesis to therapy: Controversies and perspectives. World J Gastroenterol 20: 923-942, 2014.

3. Malapelle U: USP11 role in colorectal cancer growing and metastatisation. EBioMedicine 48: 5-6, 2019.
4. Ferlay J, Steliarova-Foucher E, Lortet-Tieulent J, Rosso S, Coebergh JW, Comber H, Forman D and Bray F: Cancer incidence and mortality patterns in Europe: Estimates for 40 countries in 2012. Eur J Cancer 49: 1374-1403, 2013.

5. Rodríguez-Portal JA: Efficacy and safety of Nintedanib for the rreatment of idiopathic pulmonary fibrosis: An update. Drugs R D 18: 19-25, 2018.

6. Richeldi L, Costabel U, Selman M, Kim DS, Hansell DM, Nicholson AG, Brown KK, Flaherty KR, Noble PW, Raghu G, et al: Efficacy of a tyrosine kinase inhibitor in idiopathic pulmonary fibrosis. N Engl J Med 365: 1079-1087, 2011.

7. Crestani B, Huggins JT, Kaye M, Costabel U, Glaspole I, Ogura T, Song JW, Stansen W, Quaresma M, Stowasser S, et al: Long-term safety and tolerability of nintedanib in patients with idiopathic pulmonary fibrosis: Results from the open-label extension study, INPULSIS-ON. Lancet Respir Med 7: 60-68, 2019.

8. Collard HR, Richeldi L, Kim DS, Taniguchi H, Tschoepe I, Luisetti M, Roman J, Tino G, Schlenker-Herceg R, Hallmann C, et al: Acute exacerbations in the INPULSIS trials of nintedanib in idiopathic pulmonary fibrosis. Eur Respir J 49: 1601339, 2017.

9. Xue $\mathrm{H}$ and Tian GY: MiR-429 regulates the metastasis and EMT of HCC cells through targeting RAB23. Arch Biochem Biophys 637: 48-55, 2018.

10. Nishijima N, Seike M, Soeno C, Chiba M, Miyanaga A, Noro R, Sugano T, Matsumoto M, Kubota K and Gemma A: miR-200/ ZEB axis regulates sensitivity to nintedanib in non-small cell lung cancer cells. Int J Oncol 48: 937-944, 2016.

11. Tian X, Wei Z, Wang J, Liu P, Qin Y andZhong M: MicroRNA-429 inhibits the migration and invasion of colon cancer cells by targeting PAK6/cofilin signaling. Oncol Rep 34: 707-714, 2015.

12. Low HB and Zhang Y: Regulatory roles of MAPK phosphatases in cancer. Immune Netw 16: 85-98, 2016.

13. Gaggianesi M, Turdo A, Chinnici A, Lipari E, Apuzzo T, Benfante A, Sperduti I, Di Franco S, Meraviglia S, Lo Presti E, et al: IL4 primes the dynamics of breast cancer progression via DUSP4 inhibition. Cancer Res 77: 3268-3279, 2017.

14. Kang X, Li M, Zhu H, Lu X, Miao J, Du S, Xia X and Guan W: DUSP4 promotes doxorubicin resistance in gastric cancer through epithelial-mesenchymal transition. Oncotarget 8: 94028-94039, 2017.

15. Livak KJ and Schmittgen TD: Analysis of relative gene expression data using real-time quantitative PCR and the 2(-Delta Delta C(T)) Method. Methods 25: 402-408, 2001.

16. Wollin L, Wex E, Pautsch A, Schnapp G, Hostettler KE, Stowasser S and Kolb M: Mode of action of nintedanib in the treatment of idiopathic pulmonary fibrosis. Eur Respir J 45: 1434-1445, 2015

17. Kolb M, Richeldi L, Behr J, Maher TM, Tang W, Stowasser S, Hallmann C and du BoisRM: Nintedanib in patients with idiopathic pulmonary fibrosis and preserved lung volume. Thorax 72: 340-346, 2017.

18. Zhao YY, Feng JB, Peng J, Meng LH, Zhang HX and Wu ML: Signal mining and post-marketing evaluation of adverse drug reactions of nintedanib. Zhongguo Yiyuan Yaoxue Zazhi 39: 1655-1658, 2019 (In Chinese).

19. Januszyk P, Januszyk K, Wierzbik-Strońsk M, Boroń D and Grabarek B: Analysis of the differences in the expression of mRNAs and miRNAs associated with drug resistance in endometrial cancer cells treated with salinomycin. Curr Pharm Biotechnol: June 29, 2020. https://doi.org/10.2174/138920102166 6200629151008.

20. Lagos-Quintana M, Rauhut R, Lendeckel W and Tuschl T: Identification of novel genes coding for small expressed RNAs. Science 294: 853-858, 2001.

21. Ambros V: MicroRNA pathways in flies and worms: Growth, death, fat, stress, and timing. Cell 113: 673-676, 2003.

22. Lu J, Getz G, Miska EA, Alvarez-Saavedra E, Lamb J, Peck D, Sweet-Cordero A, Ebert BL, Mak RH, Ferrando AA, et al: MicroRNA expression profiles classify human cancers. Nature 435: 834-838, 2005.

23. Wu CL, Ho JY, Hung SH and Yu DS: miR-429 expression in bladder cancer and its correlation with tumor behavior and clinical outcome. Kaohsiung J Med Sci 34: 335-340, 2018.

24. Zhang M, Dong BB, Lu M, Zheng MJ, Chen H, Ding JZ, Xu AM and Xu YH: miR-429 functions as a tumor suppressor by targeting FSCN1 in gastric cancer cells. Onco Targets Ther 9: 1123-1133, 2016. 
25. Guo C, Zhao D, Zhang Q, Liu S and Sun MZ: miR-429 suppresses tumor migration and invasion by targeting CRKL in hepatocellular carcinoma via inhibiting Raf/MEK/ERK pathway and epithelial-mesenchymal transition. Sci Rep 8: 2375, 2018.

26. Wang F, Jiang C, Sun Q, Yan F, Wang L, Fu Z, Liu T and Hu F: Downregulation of miR-429 and inhibition of cell migration and invasion in nasopharyngeal carcinoma. Mol Med Rep 13: 3236-3242, 2016.

27. Han Y, Zhao Q, Zhou J and Shi R: miR-429 mediates tumor growth and metastasis in colorectal cancer. Am J Cancer Res 7: 218-233, 2017

28. Xiao P, Liu W and Zhou H: miR-429 promotes the proliferation of non-small cell lung cancer cells via targeting DLC-1. Oncol Lett 12: 2163-2168, 2016.

29. Wang Z, Zhu Z, Lin Z, Luo Y, Liang Z, Zhang C, Chen J and Peng P: miR-429 suppresses cell proliferation, migration and invasion in nasopharyngeal carcinoma by downregulation of TLN1. Cancer Cell Int 19: 115, 2019.

30. Ha J, Kang E, Seo J and Cho S: Phosphorylation dynamics of JNK signaling: Effects of dual-specificity phosphatases (DUSPs) on the JNK pathway. Int J Mol Sci 20: 6157, 2019. doi: 10.3390/ ijms20246157.
31. Chen P, Hutter D, Yang X, Gorospe M, Davis RJ and Liu Y: Discordance between the binding affinity of mitogen-activated protein kinase subfamily members for MAP kinase phosphatase-2 and their ability to activate the phosphatase catalytically. J Biol Chem 276: 29440-29449, 2001.

32. Muhammad T, Zhang J, Ma Y, Li Y, Zhang F, Zhang Y and Liang Y: Overexpression of a mitogen-activated protein kinase SIMAPK3 positively regulates tomato tolerance to cadmium and drought stress. Molecules 24: 556, 2019. https://doi.org/10.3390/ molecules24030556.

33. Wagner EF and Nebreda AR: Signal integration by JNK and p38 MAPK pathways in cancer development. Nat Rev Cancer 9: 537-549, 2009.

34. Raitano AB, Halpern JR, Hambuch TM and Sawyers CL: The Bcr-Abl leukemia oncogene activates Jun kinase and requires Jun for transformation. Proc Natl Acad Sci USA 92: 11746-11750, 1995.

35. Siddiqui MA and Reddy PA: Small molecule JNK (c-Jun N-terminal kinase) inhibitors. J Med Chem 53: 3005-3012, 2010.

36. Davis RJ: Signal transduction by the JNK group of MAP kinases. Cell 103: 239-252, 2000. 